\title{
Adaptive Cascade Classifier based Multimodal Biometric Recognition and Identification System
}

\author{
Ujwalla Gawande \\ Assistant Professor \\ Department of Computer Technology \\ YCCE, Maharashtra, Nagpur
}

\author{
Kamal Hajari \\ Assistant Professor \\ Department of Computer Technology \\ YCCE, Maharashtra, Nagpur
}

\begin{abstract}
Biometrics consists of techniques for uniquely recognizing humans based upon one or more intrinsic physical or behavioral traits such as Iris, fingerprint, Face and Palm geometry etc. To overcome the limitations of Unimodal biometric system, a multimodal biometric is proposed. Amongst the various fusion levels, feature level fusion is expected to offer better recognition. Feature level fusion fused the extracted feature obtained from biometric traits. The proposed system is based on feature level fusion and adaptive cascade classifier for precise and reliable multimodal recognition and identification. Verification of Genuine and imposter individual classification is done using Backpropagation neural network. The simulation results demonstrated that a multibiometric template provides better recognition performance compared to a unibiometric template and adaptive cascade classification system significantly outperforms single classifier.
\end{abstract}

\section{General Terms}

Adaptive Cascade Classifier, Feature level fusion.

\section{Keywords}

Neural network, Multimodal, single algorithmic, Multi algorithmic, Train and Test parameters and Backpropagation neural network.

\section{INTRODUCTION}

Biometrics based authentication systems is based on unique identification of measurable physical characteristics of the traits. A number of biometrics is in use by several governmental and private sectors. Such traits are like: fingerprint, face geometry, iris of the eye, voice print, hand geometry, vein patterns etc. This kind of identification cannot be readily imitated or forged by users, as these features are unique for every individual. Identification based on single trait is called as Unimodal biometrics. Though widely used, Unimodal biometric systems have variety of problems such as noisy data, intra-class variations, restricted degree of freedom, non-universality, spoof attacks, and unacceptable error rates, especially FAR(False acceptance rate) and FRR(False rejection rate). A solution to some of these problems is a fusion of two or more modalities into a single system, called as Multimodal Biometrics. In other words Multimodal Biometrics refers the combination of two or more biometric modalities in a single identification system. Multimodal biometric system is gaining more importance now days due to increase in applications requiring a high level of protection for data and services. For example; USA police utilize different biometrics modalities of criminals for detection and verifications.

Our approach is to solve these problems, by designing multimodal biometric system using iris, fingerprint, face, palm geometry. In proposed framework feature extraction technique are used for iris Blocksum, Fingerprint minutiae, palm geometry principal lines, wrinkles and ridges identified and used as features. Face detection skin mapped based feature are extracted. At identification side we apply a classifier either Probabilistic neural network (PNN) or Radial basis neural network (RBF) and adaptive Cascade classifier which are based on mean and variance values. It will classify query vector which in turn gives us decision i.e. if user is accepted or rejected. For verification, we used Backpropagation neural network to get the result whether user is genuine or imposter. One more important parameter is response time, as user cannot wait forever for authentication. The response time of the proposed system is satisfactory due to selection of classifiers in this approach which requires fraction of seconds to classify a user. The most important metrics of biometric recognition system is FAR which should be less in any application areas. Our system guarantees low FAR as well as FRR.

\section{LITERATURE SURVEY}

Traditional methods for personal identification are based on what a person possesses (a physical key, ID card etc.) or what a person knows (a secret password, etc.).These methods have disadvantages such as keys may be lost, ID cards may be forged, and passwords may be forgotten. Human users have a tough time remembering long cryptographic keys. Hence, researchers, for so long, have been examining ways to utilize biometric features of the user instead of a memorable password or passphrase. In recent Years, biometric personal identification is receiving growing interests from both academia and industry [1]. This is because biometrics are required to carry but they are always with us, and there is no issue of theft or forgetting.A Unimodal system focuses on use on a single biometric modality and relies on it for result/performance. Unimodal biometric systems have variety of problems such as noisy data, intra-class variations, restricted degree of freedom, non-universality, spoof attacks, and unacceptable error rates, especially high FAR and FRR [2]. Iris is one of the popular biometric traits. The probability of finding two people with identical iris patterns is considered to be approximately 1 in 1052 (population of the earth is of the order 1010). Not even one-egged twins or a future clone of a person will have the same iris patterns. The iris is considered to be an internal organ because it is so well 
protected by the eyelid and the cornea from environmental damage. It is stable over time even though the person ages. Same goes for Fingerprint; it is the oldest and most researched form of biometric. The probability of finding two people with identical fingerprint patterns is considered to be approximately 6 in total world population. [3][4]. Y. Gao et al.[5] proposed a unique method for face recognition by mapping line edges of a face. Xiangqian $\mathrm{Wu}$ et al. [6] proposed a novel method for extracting feature vector of palm geometry by determining the stable lines, orientation angle, ridges. Multimodal biometrics refers the combination of two or more biometric modalities in a single identification system. They are more reliable than their Unimodal counterparts, as they combine the results of two or more biometric modalities. The most compelling reason to combine different modalities is to improve the recognition rate. [7]

Very few researchers have tried to fuse iris and Fingerprint as a multimodal biometric system though both of them are proven as reliable techniques individually. Asim Baig et al.[8] proposed a Fingerprint and iris fusion using single hamming distance matcher. They have fused the individual score at matching levels and obtained a low FAR and FRR. Feten Besbes et al. [9] proposed the fusion of iris and Fingerprint at decision level using AND operation. A. Jamir et al. [10] proposed Fingerprint and iris fusion and utilize adaptive rank level fusion at verification stage and found the enhanced performance of $95.6 \%$ for multimodal biometric, face $83.5 \%$, Fingerprint $85.2 \%$ and iris $87.6 \%$. Afore mentioned research by various authors tried to make their decision of multimodal system at the decision level. A variety of articles can be found, which propose different approaches for multimodal biometric systems. Multimodal biometric systems are based on different biometric features and/or introduce different fusion algorithms for these features. Many researchers have demonstrated that the fusion process is effective, because fused scores provide much better discrimination than individual scores [11]. Such results have been achieved using a variety of fusion techniques. Donald E. Maurer and John P. Baker et al. [12] have presented fusion architecture based on Bayesian belief networks. Minutiae feature extraction method for fingerprint images give better results than any other feature extraction algorithm.[13] A reliable and efficient fusion algorithm is heart of the thesis. Choosing between the types of fusion is also a very important decision while designing a multimodal biometric system. The feature level fusion is more efficient and responsive as compared to other types of fusion [11]. Muhammad Khurram Khana and Jiashu Zhanga presented an efficient multimodal face and fingerprint biometrics authentication system on space-limited tokens, e.g. smartcards, driver license, and RFID cards [14]. A class dependence feature analysis technique based on Correlation Filter Bank (CFB) technique for efficient multimodal biometrics fusion at the feature level is presented by Yan Yan and Yu-Jin Zhang [15]. In CFB, the overall original correlation outputs were optimized and unconstrained correlation filter were trained for a specific modality. As a result variation between modalities was considered and the useful information in various modalities was completely utilized. Previous experimental outcome on the fusion of face and palm print biometrics proved the advantage of their technique.

Classifiers are needed to perform matching of query with database. A wide range of classifiers are available. A probabilistic neural network (PNN) classifier maps any input pattern to a number of classifications to be forced into a more general function approximate. A PNN is an implementation of a statistical algorithm called kernel discriminate analysis in which the operations are organized into a multilayered feed forward network with four layers, input layer, pattern layer, summation layer and output layer [16]. A radial basis function (RBF) neural network is trained to perform a mapping from $\mathrm{m}$ $\mathrm{x} \mathrm{n}$ dimensional input space to an $\mathrm{n}$ dimensional output space. RBFs can be used for discrete pattern classification, function approximation, signal processing, control, or any other application which requires a mapping from an input to an output [17]. A support vector machine (SVM) is a classifier that is used to analyze data and recognize patterns, used for classification and regression analysis. The standard SVM takes a set of input data and predicts, for each given input, which of two possible classes that is person is Genuine or Imposter [18]. The Back Propagation neural network (BPNN) belong to the multi-layers feed-forward neural networks, which have one input layer, one output layer, and one or more hidden layers. Usually, we use the BP neural networks with 3 layers. In the image recognition, the number of the input layers cells is equal to the dimension of the input pattern and the number of the output layers cells is equal to the number of the objects to be recognized. It is difficult to determine the number of the hidden layers cells. The steps of training can be reduced when we increase the number of the hidden layers cells, however, the redundancies of the networks would be increased. Generally, we used the methods by trying to determine the hidden layers cells number. With respect to the limitation of the standard BP networks arithmetic, the L-M arithmetic [19] is more preferable.

\section{PROPOSED WORK}

Proposed framework is model into two parts identification and verification. In identification phase, the classifiers used are PNN and RBFNN, for obtaining the precision in decision of adaptive cascade classifier. In verification phase, BPNN classify user as Genuine or Imposter. These two processes are described as follows.

\section{IRIS AND FINGERPRINT PRE- PROCESSING}

Iris recognition is the most precise and fastest Authentication method. The first step in iris recognition is the preprocessing step. This step is further divided into different steps i.e. Image acquisition, localization, segmentation and normalization. Image acquisition is the first step, which captures the Iris Image. In localization step, acquired iris image has to be preprocessed to detect the iris, which is an annular portion between the pupil (inner boundary) and the sclera (outer boundary).The task consists of localizing the inner and outer boundaries of the iris, both are circular, but the problem lies in the fact that they are not co-centric. It is necessary to calculate the two circles parameters separately. The first step in iris localization is to detect pupil which is the black circular part surrounded by iris tissues. The center of pupil can be used to detect the outer radius of iris patterns. The important step involved is pupil detection (inner boundary). Iris image is converted into grayscale to remove the effect of illumination. As pupil is the largest black area in the intensity image, its edges can be detected easily from the binaries image by using suitable threshold on the intensity image. But the problem of binarization arises in case of persons having dark iris. Thus the localization of pupil fails in such cases. Hough 
Transformation method is used to overcome these problems. Circular Hough Transformation is used to detect the pupil. The basic idea of this technique is to find curves that can be parameterized like straight lines, polynomials, circles, etc., in a suitable parameter space. The Hough transform is an image processing technique which is effective in determining the parameters of simple geometric shapes. The circular Hough transformation is applied on the iris image, to compute the radius and center. For Outer iris localization (outer boundary), External noise is removed by blurring the intensity image. But too much blurring may dilate the boundaries of the edge or may make it difficult to detect the outer iris boundary, separating the eyeball and sclera. This contrast enhanced image is used for finding the outer iris boundary by drawing concentric circles of different radius from the pupil center and the intensities lying over the perimeter of the circle. Among the iris circles, the circle having a maximum change in intensity with respect to the previous drawn circle is the iris outer boundary. Segmentation follows localization to separate eyelid and eyelashes portion from actual iris part. Circular Hough transforms method increase the efficiency of circle detection process and speed up execution. The success of segmentation depends on the quality of eye Images. The persons with darkly pigmented irises will present very low contrast between the pupil and iris region, if imaged under natural light, making segmentation more difficult. Canny edge detection is used generate edge map of an image. It is multistage algorithm to compute the wide range of edges of images. starts with linear filtering to compute the gradient of the image intensity distribution function and ends with thinning and Thresholding to obtain a binary map of edges. One significant feature of the canny operator is its optimality in handling noisy images as the method bridges the gap between strong and weak edges of the images by connecting the weak edge in the output only if they are connecting to strong edges. Therefore, the Edge will probably be the actual ones. Then these iris segmentation algorithms achieve high performance on CASIA iris database images. Then next most important step in preprocessing of iris is Normalization in which, Image is captured in different size, for the same person also size may vary because of the variation in illumination and other factors. The normalization process will produce iris regions, which have the same constant dimensions, so that two photographs of the same iris under different conditions will have Characteristic features at the same spatial location. The Daugman rubber sheet modal is used; in this circular image is transformed into rectangular form with the size $20 \mathrm{x}$ 240 i.e. vertically 20 and horizontally 240 pixels.

Fingerprint recognition includes different steps. The first step is the preprocessing step. This step is further divided into different steps i.e. Image acquisition, segmentation and normalization. Image acquisition is the first step, which captures the Fingerprint Image. In this fingerprint image is capture by the sensor, it is not good to directly used for feature extraction so we apply several preprocessing on the fingerprint image to enhance their features and make it as a quality image. In Segmentation, the foreground region is separated from the background regions. The foreground regions correspond to the clear fingerprint area containing the ridges and valleys; this is the area of interest. The background corresponds to the regions outside the borders of the fingerprint area, which do not contain any valid Fingerprint information. Cutting or cropping out the region that does not contain valid information minimizes the number of operations on fingerprint image. The background regions of a fingerprint image generally exhibit a very low grey-scale variance value, whereas the foreground regions have a very high variance. Hence, a method based on variance Thresholding can be used to perform the segmentation. Segmentation followed by Normalization step in this process range of pixel intensity values is adjusted. Normalization is sometimes called contrast stretching. It is referred to as dynamic range expansion. The purpose of dynamic range expansion in the various applications is usually to bring the image, or other type of signal, into a range that is more familiar or normal to the senses, hence the term normalization. Normalization is a linear process. If the intensity range of the image is 10 to 180 and the desired Range is 0 to 255 the process entails subtracting 10 from each of pixel intensity, making the range 0 to 120 . Then intensity of each pixel is multiplied by 255/120, making the range 0 to 255 . To enhance the normalized image Histogram equalization (HE) is a very common technique for enhancing the contrast of an image. Here, the basic idea is to Map the gray levels based on the probability distribution of the input gray levels. HE flattens and stretches the dynamic range of the images histogram resulting in overall contrast improvement of the image. It will improve the recognition rate.

\section{FACE AND PALM GEOMETRY PRE- PROCESSING}

Face recognition is somehow critical task; it is perform in several steps as Face image acquisition, Enhancement, Feature extraction and data normalization. Image acquisition is first step in which Face image is capture. To enhance the image filters are used, which will help in extracting the rich feature. Due to this recognition rate and accuracy is increased. Palm geometry preprocessing can be done in parts, In which palm edges are mapped, orientation angle, Termination and Bifurcation points are identified and also the position, direction, and amount of stretching of a palm may vary so that even if palm prints may have little rotation and translation it will not cause any problem.

\section{RECOGNITION AND IDENTIFICATION OF MULTIMODAL BIOMETRIC}

Multimodal recognition and identification can be done using in two module Identification phase and verification phase using different classifiers.

\subsection{FEATURE EXTRACTION}

Iris features are extracted using Blocksum method, in which normalized image is divided in blocks and entropy value of each block is computed, it is unique for each block that will be stored as features vector. Fingerprint features are extracted using Minutiae technique, in which normalized fingerprint image bifurcation and termination points are identified with their orientation angle that will be stored as feature vector. To obtain these points, certain steps are performs such as Binarization, Thinning, Minutiae point Extraction and Orientation and Region of interest (ROI). Face features are extracted using skin mapped technique, in which extracted face parameters are stored as feature vector. Palm geometry features using principle line, strucal, edge mapping parameter 
are stored as feature vector. These extracted features will be fused using Fusion technique. To obtained Multimodal Biometric Template.

\subsection{FUSION TECHNIQUE}

Feature level fusion is expected to provide high accuracy rate as it works on row data as compared to higher level of fusion. Iris and fingerprint feature vectors complement each other to provide higher recognition rate. We proposed a novel feature level fusion method using convolution theorem which reduces false acceptance and false rejection rates effectively. Let $F_{1}=\left[\begin{array}{lllll}x_{1} & x_{2} & x_{3} & \ldots & x_{n}\end{array}\right]$, the feature vector of iris using Blocksum method. Let $\mathrm{F}_{2}=\left[\begin{array}{lll}y_{1} & y_{2} & y_{3} \ldots y_{n}\end{array}\right]$, the feature vector of fingerprint using Minutiae method. Same for Face $F_{3}=\left[z_{1} z_{2}\right.$ $\left.z_{3} \ldots z_{n}\right]$, and palm geometry $F_{4}=\left[a_{1} a_{2} a_{3} \ldots a_{n}\right]$. Feature level fusion is done to obtained the Multimodal Biometric template, this process is divided into parts as Iris and Fingerprint features are fused and In other side Face and Palm geometry feature are fused using the convolution theorem by convolving $F_{1}$ on $F_{2}$ which will result in output vector $I_{1}=\left[i_{1}\right.$ $\left.i_{2} i_{3} \ldots i_{n}\right]$.and $F_{3}$ on $F_{4}$ which will result output vector $I_{2}=\left[j_{1}\right.$ $\left.j_{2} j_{3} \ldots j_{n}\right]$. Then multiply each element in $I_{1}$ and $I_{2}$ with same index positions. To obtained the fused Multimodal template $M_{1}=\left[m_{1} m_{2} m_{3} \ldots m_{n}\right]$.
Convolution theorem works on multiplying each of the extracted Features and summing the pixels in the neighborhood. Generated Multimodal fused Template further goes for the identification and Verification process.

\subsection{CLASSIFICATION USING PNN}

A probabilistic neural network (PNN) is predominantly a classifier. It can map any input pattern to a number of classifications, to be forced into a more general function approximate. A PNN is an implementation of a statistical algorithm called kernel discriminate analysis in which the operations are organized into a multilayered feed forward network with four layers as: Input layer, Pattern layer, Summation layer and Output layer. In Input Layer Multimodal fused vector template pass as input pattern for the neural network. In Pattern Layer training and classification of features is done. It classifies an individual features vector to a class. In Summation Layer, classified vector of each subject consist of several feature vector that can be com-bine into a single unit because they are a nearer to each other. In the Output Layer, query vector is match with the classified feature vector and appropriate match vector is shown in the output.

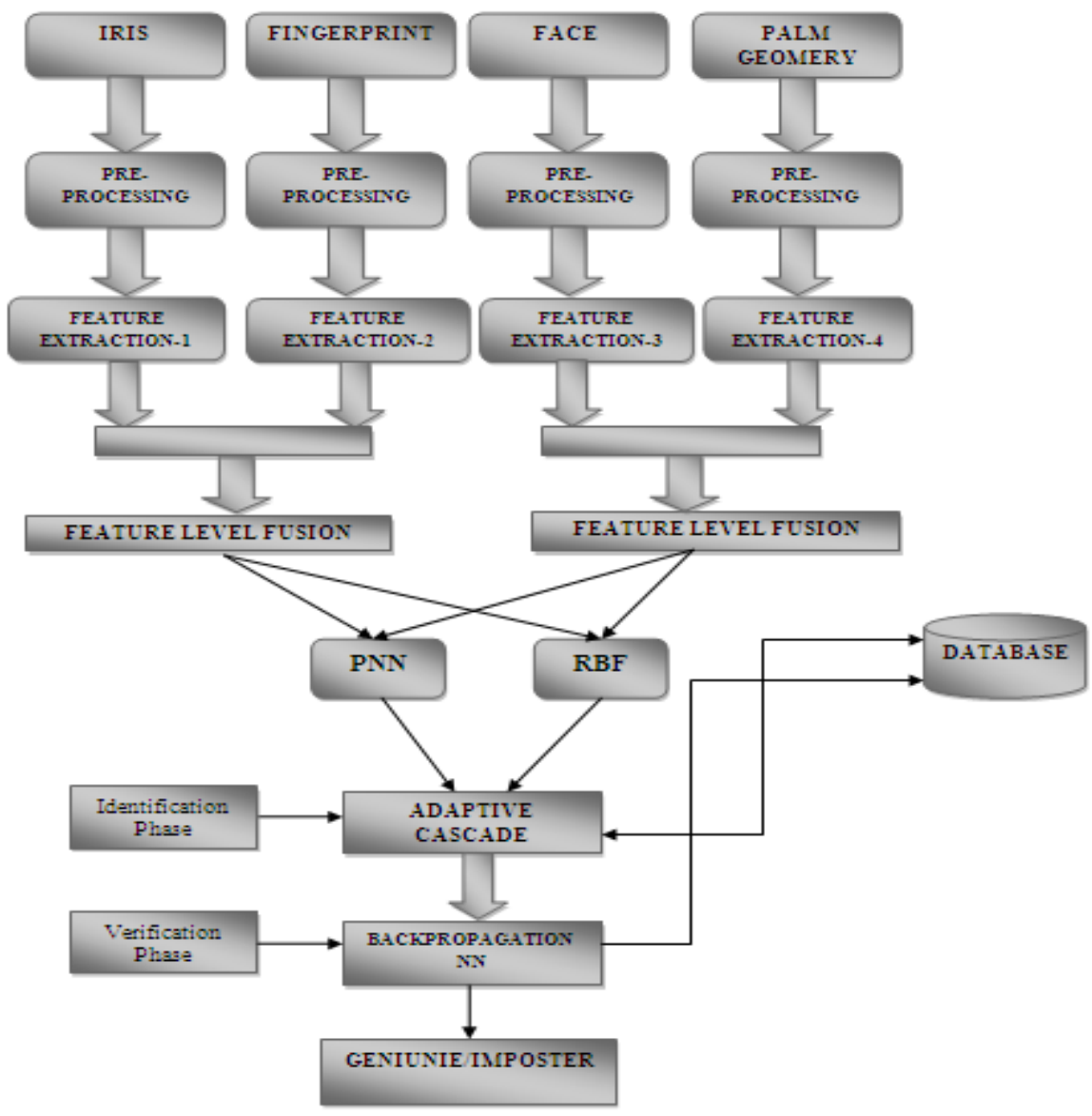

Figure 1: Overall Architecture of Proposed System 


\subsection{CLASSIFICATION USING RBF}

A radial basis function (RBF) neural network is more efficient than the PNN classifier, trained to perform a mapping from $\mathrm{m}$ $\mathrm{x} \mathrm{n}$ dimensional input space to an $\mathrm{n}$-Dimensional output space. RBFNN can be used for discrete pattern classification, function approximation, signal processing, control, or any other application which requires a mapping from an input to an output. The RBFNN classifier is three layer architecture as; Input Layer, Middle Layer and Output Layer. In Input Layer Multimodal fused vector template pass as input pattern for the neural network. In Middle Layer training and classification of features vector is done. It classifies an individual features vector to a class. The classified vector are grouped which are nearer to each other belongs to their respective subject. It is also called as hidden layer; the hidden layer applies an activation function which is a function of the Euclidean distance between the input and an m-dimensional prototype vector. In Output Layer, the query vector is match with the classified trained vector and appropriate match vector is shown in the output.

\subsection{ADAPTIVE CASCADE CLASSIFICATION}

Adaptive Cascade classification based on the principles of mean and variance values, which is computed for each query and database Multimodal fused feature vector and training is done with respect to the decision of neural network and network is tuned using mean and variance instead of weight which will give result as accepted and rejected. Then after identifying the individual it is go towards the verification phase.

Table 1: FAR, FRR and GAR

\begin{tabular}{|c|c|c|c|}
\hline Classifiers & FRR\% & FAR\% & GAR\% \\
\hline PNN & $3 \%$ & $2 \%$ & $97 \%$ \\
\hline RBFNN & $4 \%$ & $3 \%$ & $96 \%$ \\
\hline $\begin{array}{c}\text { Adaptive } \\
\text { Cascade } \\
\text { Classifier }\end{array}$ & $1.2 \%$ & $2 \%$ & $98.8 \%$ \\
\hline BNN & $4 \%$ & $3.7 \%$ & $96 \%$ \\
\hline
\end{tabular}

Table 1: Multimodal Training and Testing Set

\begin{tabular}{|c|c|c|c|}
\hline $\begin{array}{c}\text { Classifier } \\
\text { S }\end{array}$ & Training mages & Testing Images & Total \\
\cline { 1 - 1 } PNN & & & \\
\cline { 1 - 1 } RBFNN & & & \\
\cline { 1 - 1 } $\begin{array}{c}\text { Adaptive } \\
\text { Classifier }\end{array}$ & 400 & 100 & 500 \\
\cline { 1 - 2 } BNN & & & \\
\hline
\end{tabular}

\subsection{VERIFICATION PHASE}

In verification phase Backpropagation neural network (BNN) is used which classify user to as Genuine and imposter, depicted briefly as follows.

\subsection{BACKPROPAGATION NEURAL NETWORK}

$\mathrm{BNN}$ is used for verification of user. In which user is classify as Genuine/Imposter. Training process can be performs in several steps, It consist of input parameter as Grouping of Training parameter as (Group of (G and I)). neural network trained the fused Multimodal Template using these parameter and take Adaptive Cascade classifier decision vector as query vector which will be used as Testing pattern give decision in output as 'G' and 'I'.

\section{EXPERIMENT RESULTS}

Experiment result perform on several samples of CASIA Iris Database, Fingerprint samples are collected in own college and Face and palm geometry database is the standard database. And after performing several training and Testing of different Neural networks, finally Adaptive Cascade classifier performance is more satisfactory as compare to single classifier and proposed framework FAR and FRR is nearer to zero percent.

\section{CONCLUSION}

Biometric systems are widely used to overcome from the drawbacks of the traditional methods of authentication. Unimodal biometric systems fail in some cases and hence cannot be used for high security applications. Thus we proposed a multimodal biometric approach. In which feature level fusion increased the recognition rate using novel method. Experimental result shows that multimodal systems perform better as compared to Unimodal biometrics. We are not challenging the existing work of the researchers but this proposed technique can be an effective alternative to the existing methods. Proposed Multimodal based Adaptive classifier framework gives higher accuracy percentage up to 98.8\% and precision in results and give Flexible approach to a biometric Identification and verification system.

\section{REFERENCES}

[1] A. K. Jain, A. Ross, and S. Prabhakar, "An Introduction to Biometric Recognition", IEEE Transactions on Circuits and Systems for Video Technology, Special Issue on Image and Video Based Biometrics, vol. 14, no. 1, pp. 4-20, January 2004.

[2] Libor Masek, A. Kumar and A. Passi, "Comparison and Combination of Iris Matchers for Reliable Personal Authentication", Journal of Pattern Recognition, ScienceDirect,vol. 43, no.3, pp. 1016-1026, March 2010.

[3] N. K. Ratha, R. M. Bolle , V. D. Pandit and V. Vaish, "Robust Fingerprint Authentication using Local Structural Similarity", Proceedings of 5th IEEE Workshop on Application of Computer Vision (WACV), Santa Barbara, CA, pp. 29-34, December 2000. 
[4] M. Nabti, L. Ghouti and Ahmed Bouridane, "An Efficient and fast Iris Recognition System based on a Combined Multiscale Feature Extraction Technique", Journal of the Pattern Recognition, ScienceDirect, vol. 41, no. 3, pp. 868-879, March 2008.

[5] Y. Gao and M. Leung, "Face recognition using line edge map", IEEE Transactions on Pattern Analysis and Machine Intelligence, vol. 24, no. 6, pp. 764-779, Jun 2002.

[6] Xiangqian Wu, Zhang D. and Kuanquan Wang, "Palm Line Extraction and Matching for Personal Authentication",, IEEE Transactions on Pattern Analysis and Machine Intelligence, vol. 36, no. 5, pp. 978-987, September 2006.

[7] Teddy Ko, "Multimodal Biometric Identification for Large User Population Using Fingerprint, Face and Iris Recognition", Proceeding of $34^{\text {th }}$ Applied Imagery and Pattern Recognition Workshop (AIPR05), IEEE Computer Society Washington, DC, USA, pp. 218- 223, December 2005.

[8] Asim baig, Ahmed Bouridane, Fatih Kurugollu and Gang $\mathrm{Qu}$, "Iris Fusion based identification system using a single hamming distance matcher", International Journal of Bio-Science and Bio-Technology (IJBSBT), vol. 1, no. 1, pp. 46-58, December 2009.

[9] Besbes, Hanene Trichilli and Basel Solaiman, "Multimodal Biometric System Based on Fingerprint Identification and Iris Recognition", IEEE Transactions on Image Processing, vol. 5, pp 947-952, 2006.

[10] A. jameer Basha, V. Palanisamy, T. Purusotoman, "Fast multimodal biometric approach using dynamic Fingerprint authentication and enhanced iris Features", IEEE International Conference on Computational Intelligence and Computing research(ICCC), Coimbatore, pp. 1-8, 28-29,December 2010.
[11] Arun Ross, Rohin Govindrajan, "Feature level fusion in biometric system", "Feature level fusion in Biometric system", West Virginia University, Morgantown, pp. 12, 2009

[12] Donald E. Maurer and John P. Baker," Fusing multimodal biometrics with quality estimates via a Bayesian belief network“, Pattern Recognition, vol. 41, no. 3, pp. 821-832, March 2008.

[13] Kulwinder Singh, Kiranbir Kaur, Ashok Sardana, "Fingerprint Feature Extraction", International Journal of Computer Science and Technology (IJCST), vol. 2, no. 3, September 2011.

[14] M. Khurram Khana and Jiashu Zhanga, "Multimodal face and fingerprint biometrics authentication on spacelimited tokens ", Nerocomputing, , vol. 71, no. 13-15, pp. 3026-3031, August 2008.

[15] Yu-Jin Zhange and Y. Yan, "Multimodal biometrics fusion using correlation Filter Bank", Proceeding of the 9th International Conference on Pattern Recognition, pp. 1-4, Tampa, FL, 2008.

[16] Donald F. Specht, "Probabilistic neural network", Proceeding of conference on neural networks, vol. 3, no. 1, pp 109-118, 1990

[17] M. Birgmeier, "A fully Kalman-trained radial basis function network for nonlinear speech 11 modeling", IEEE International Conference on Neural Networks, Perth, Western Australia, pp. 259-264.1995.

[18] Fahmy, Atiya, Raafat, Elfouly, "Biometric Fusion using Enhanced SVM classification", Intelligent Information Hiding and Multimedia Signal Processing (IIHMSP), IEEE, Harbian, 15-17, August 2008.

[19] J. Ni and L. Shao,” The Observational Data Analysis and Processing by Neural Network", Journal of Lianyungang College of Chemical Technology, vol.13, no.4, pp.34-36, December 2000. 